\title{
Efficacy of foot orthoses for the treatment of plantar heel pain: a systematic review and meta-analysis
}

\author{
Nadine Rasenberg, ${ }_{1}^{1}$ Henrik Riel, ${ }^{2}$ Michael S Rathleff, ${ }^{2}$ Sita M A Bierma-Zeinstra, ${ }_{1}^{1}$ \\ Marienke van Middelkoop ${ }^{1}$
}

- Additional material is published online only. To view please visit the journal online (http://dx.doi.org/10.1136/ bjsports-2017-097892).

'Department of General Practice, Erasmus Medical Center, Universitair Medisch Centrum, Rotterdam, The Netherland

${ }^{2}$ Research Unit for General Practice in Aalborg, Department of Clinical Medicine, Aalborg University, Aalborg, Denmark

\section{Correspondence to}

Dr Nadine Rasenberg, Department of General Practice, Erasmus Medical Center, Universitair Medisch Centrum, Rotterdam 2040, CA, The Netherland; n.rasenberg@erasmusmc.nl

Accepted 22 December 2017 Published Online First 19 March 2018
ABSTRACT

Background Plantar heel pain (PHP) is common. Foot orthoses are often applied as treatment for PHP, even though there is little evidence to support this.

Objective To investigate the effects of different orthoses on pain, function and self-reported recovery in patients with PHP and compare them with other conservative interventions.

Design Systematic review and meta-analysis. Data sources A systematic literature search was conducted in MEDLINE, EMBASE, Cochrane Central Register of Controlled Trials, Web of Science, CINAHL and Google Scholar up to January 2017.

\section{Eligibility criteria for selecting}

studies Randomised controlled trials comparing foot orthoses with a control (defined as no intervention, sham or other type of conservative treatment) reporting on pain, function or self-reported recovery in patients with PHP.

Results Twenty studies investigating eight different types of foot orthoses were included in the review. Most studies were of high quality. Pooled data from six studies showed no difference between prefabricated orthoses and sham orthoses for pain at short term (mean difference (MD) of $0.26(95 \% \mathrm{Cl}-0.09$ to 0.60$)$ ). No difference was found between sham orthoses and custom orthoses for pain at short term (MD 0.22 (95\% Cl -0.05 to 0.50$)$ ), nor was there a difference between prefabricated orthoses and custom orthoses for pain at short term (MD $0.03(95 \% \mathrm{Cl}-0.15$ to 0.22$))$. For the majority of other interventions, no significant differences were found.

Conclusions Foot orthoses are not superior for improving pain and function compared with sham or other conservative treatment in patients with PHP. PROSPERO registration number CRD42015029659.

\section{INTRODUCTION}

Plantar heel pain (PHP) is a common cause of foot pain in both primary and secondary care. ${ }^{1-3}$ PHP accounts for approximately 11\%-15\% of all foot symptoms requiring medical attention in adults and for $8 \%-10 \%$ of all running-related injuries. $^{45}$

PHP is a clinical diagnosis, which is characterised by pain over the anteromedial aspect of the inferior heel that tends to increase after periods of inactivity or during weight-bearing activities. ${ }^{6}$ Patients often report decreased quality of life as their heel pain prevents them from performing every day activities or participate in exercise or sport. ${ }^{78}$ The large impact on everyday life results in a strong desire in patients for an effective treatment. ${ }^{9}$ Even though the clinical course is considered favourable as previous literature found complete remission of symptoms in $80 \%$ of patients within 12 months. ${ }^{6}$ However, other findings suggest that more than $40 \%$ of patients still have symptoms 2 years after diagnosis. ${ }^{10}$

The most commonly prescribed treatments for PHP include footwear modification, taping, stretching exercises anti-inflammatory agents; extracorporal shock wave therapy; strengthening exercises and cortisone injections. ${ }^{6}$ 11-13 Despite a high number of randomised controlled trials (RCTs), there is still a lack of consensus on which treatments are most effective. This leaves the clinician with uncertainty on which treatment they should prescribe to decrease pain and improve function in their patients. ${ }^{11} 14$

Foot orthoses for PHP have been frequently studied in the literature and are often applied. ${ }^{11}$ 15-17 Though a Cochrane review from 2008 on the effectiveness of custom-made orthoses on foot pain concluded that there is insufficient evidence from high-quality trials to support the effectiveness of these orthoses in patients with PHP. ${ }^{17}$ Since this review, many new studies have investigated the effectiveness of orthoses in patients with PHP and a new synthesis of the literature is needed to guide the clinical decision process. The objective of this review is to investigate the effect of different foot orthoses on pain, function and self-reported recovery in patients with PHP and compare them with other conservative interventions.

\section{METHODS}

A systematic literature search was conducted in MEDLINE, EMBASE, Cochrane Central Register of Controlled Trials, Web of Science, CINAHL and Google Scholar up to January 2017 (online supplementary file 1). Additionally, reference lists of included articles were screened for relevant articles. Two review authors (NR, HR) independently screened all titles and abstracts. Full-text articles were obtained for those citations that were thought to fulfil all predetermined inclusion criteria and were screened independently by the same authors. If consensus regarding inclusion could not be reached by the two reviewers, a third reviewer (MvM) was consulted.

\section{Selection of full-text articles}

In order to be included in the systematic review, a study had to fulfil the following inclusion criteria. 
Type of studies

RCTs written in English, Danish, Dutch, German or French examining the effectiveness of treatment with foot orthoses in patients with PHP were eligible.

\section{Type of participants}

Adults and adolescents suffering from acute or chronic PHP were included. There were no limitations regarding age, sex, setting or duration of complaints. In the literature, inconsistency in terminology for PHP exists. Studies were included if they used one or more of the following criteria in the inclusion of their study population:

- tenderness or pain in the inferior heel, the medial calcaneal tuberosity at the plantar aponeurosis or the insertion of the plantar fascia on the calcaneus during rest, exercise or palpation

- clinical evidence of PHP: pain provoked when taking the first few steps in the morning or after a period of rest, increased pain at the commencement of weight bearing.

Studies including participants with a diagnosis of a different foot pathology were excluded.

\section{Type of outcomes}

Studies reporting on pain, function or recovery were included. There were no restrictions on the type of measurement tool used or duration of the intervention.

\section{Type of interventions}

Studies comparing at least one type of foot orthosis with another type of non-surgical treatment, a control group (sham or usual care) or a different type of foot orthosis were included. Night splints were not considered to be foot orthoses. The orthoses could be prefabricated, custom made or sham.

\section{Risk of bias assessment}

The risk of bias assessment was conducted using the 12 criteria recommended by the Cochrane Back Review Group. These criteria were evaluated by two researchers independently (NR and HR). The 12 criteria were answered with 'yes', 'no' or 'unclear' for each article. Differences between the two assessors were solved by consensus. Studies with six or more positively answered questions were considered to have a low risk of bias. ${ }^{18}$

\section{Data extraction}

Two reviewers (NR and HR) independently extracted data using a standardised data extraction form and compared the extracted data. Any inconsistencies were discussed between the reviewers and unresolved disagreements were solved by involving a third author $(\mathrm{MvM})$. The following data were extracted: general study information (ie, author names, country of origin), participants (ie, age, sex, duration of complaints, nature of the complaint, inclusion/exclusion criteria) and intervention characteristics (ie, description of the experimental and control interventions, co-interventions if used), compliance, duration of follow-up, withdrawals and outcome measures. Data related to pain, function and recovery were assessed, and values of all follow-up measurements were extracted $(\mathrm{N}$, mean, $\mathrm{SD}, \mathrm{N}$, median, IQR or $\mathrm{N}$ and proportion recovery). The follow-up time intervals were defined as short term (up to 3 months), midterm (from 3 to 12 months) and long term (after 12 months). If data were not available from tables or the result section, the authors of the study in question were contacted. When possible, results from the intention-to-treat population were extracted. In case of multiple outcome measures, the outcome that was used the most in the included studies was used.

\section{Data synthesis}

When possible, the mean and SD at follow-up were used. If a study only reported the mean difference (MD) as compared with baseline, the point estimate was calculated by subtracting the MD from the mean at baseline and the SD was carried forward from baseline. All outcome scores were converted to scales ranging from 0 to 100, where necessary. Review Manager V.5.2 was used for analysis, and treatment effects were expressed in MD for continuous data and the OR or relative risk (RR) for dichotomous data, including 95\% CIs. When data were considered clinically homogeneous, statistical pooling was performed according to a random effects model and standardised mean differences (SMD) were reported. If outcomes used in pooling had different directionality (eg, lower scores reflecting better or worse outcomes), all were set to the same direction. Heterogeneity in the pooled data was calculated with the $\mathrm{Q}$ test. When data were considered heterogeneous $\left(I^{2}>70 \%\right)$, descriptive statistics (MD, OR or RR) were calculated. If these were already presented by the authors, these data were used in the tables.

\section{Review of protocol}

The review protocol was written a priori and registered in PROSPERO (CRD42015029659).

\section{RESULTS}

The search identified 1766 articles of which 895 unique articles were identified after duplicates were removed. A total of 857 articles were excluded after screening title and abstract, and an additional of 18 articles were excluded after the full-text screening. Finally, 20 RCTs met the inclusion criteria and were included in this review (figure 1). Twelve authors were contacted in order to obtain more data, ${ }^{19-30}$ of which six authors were able to provide us with the required information. ${ }^{19-212729} 30$

\section{Risk of bias in the included studies}

The initial agreement between the two authors who performed the risk of bias assessment was $92 \%$. A total of 12 studies had a total score of 6 or higher and were considered to have a low risk of bias ${ }^{151619-2124} 2730-34$ (table 1). Reasons for a high risk of bias were often an unclear or inadequate method of randomisation, no allocation concealment, no blinding of patient, care provider or outcome assessor.

\section{Characteristics of the included studies}

The number of patients included in the studies ranged from 17 to 255 , with a total of 1756 patients with PHP. Follow-up of the studies ranged from 3 to 52 weeks. Five studies were performed in a hospital setting. ${ }^{16} 19242634$ and two studies in a primary care setting. ${ }^{29} 32$ For three studies, the setting was unknown. ${ }^{23} 3035$ The remaining 11 studies were set in different types of clinics for podiatric care. ${ }^{1520-2225272831333637}$ Four studies also recruited patients in the open population. ${ }^{15252734} \mathrm{~A}$ total of eight different types of orthoses and ten different types of other control interventions such as stretching exercises, corticosteroid injection or change in shoes were studied. Three studies used a sham intervention as a control online supplementary file $2 .{ }^{152127}$ 


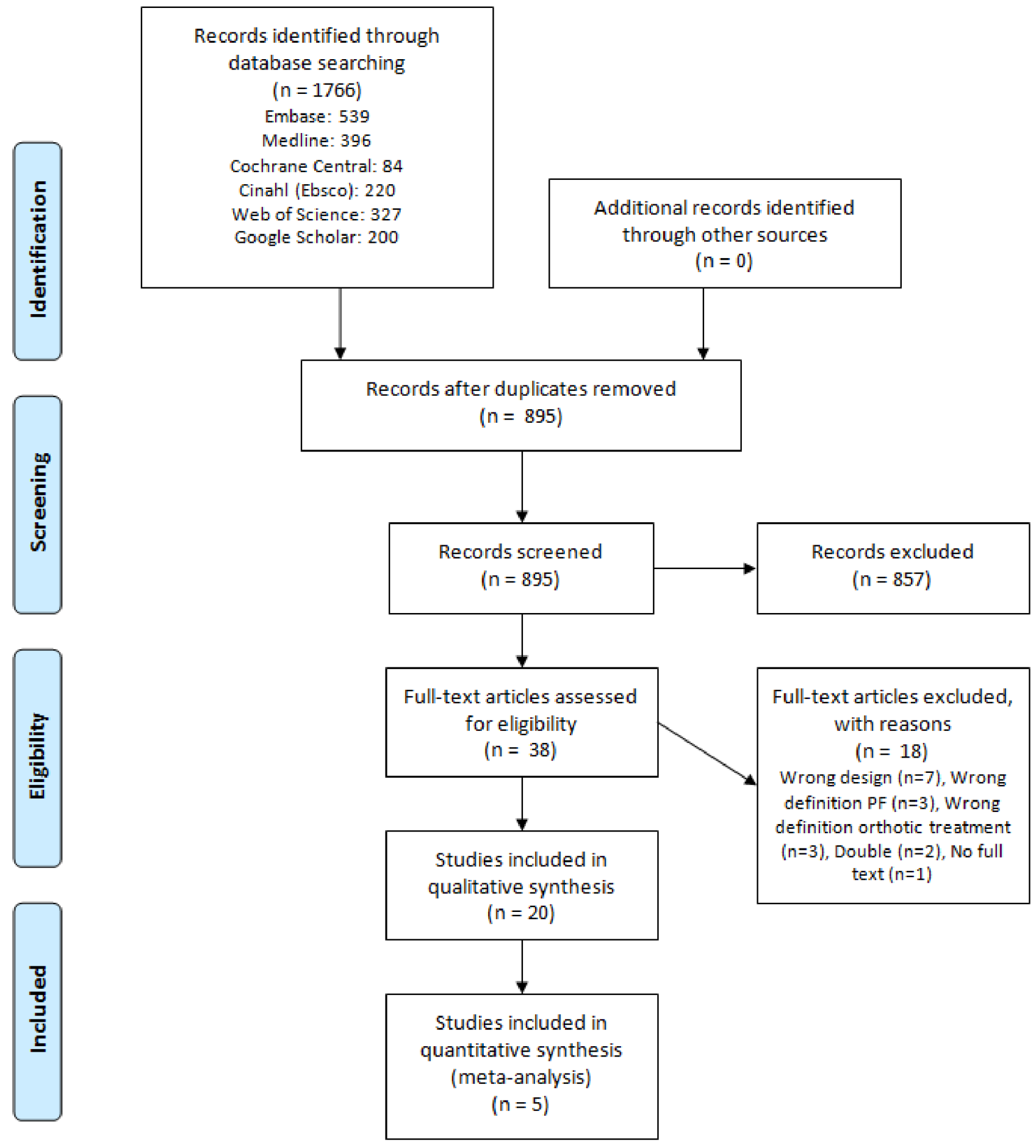

Figure 1 Flowchart of the inclusion and exclusion of articles in this review.

\section{Comparison of different types of foot orthoses}

A summary of all results per study is given in online supplementary file 3 .

\section{Prefabricated versus custom-made orthosess}

Five studies compared prefabricated orthoses with custommade orthoses. ${ }^{15} 16202137$ All studies provided data that could be used for pooling. No difference was found on short-term pain (SMD $0.03(95 \% \mathrm{CI}-0.15$ to 0.22$)$ ) between prefabricated and custom-made orthoses (online supplementary file
4). These studies were considered statistically homogeneous with an $\mathrm{I}^{2}$ of $0 \%$. Two studies with a low risk of bias reported on short-term function. Pooled results showed no difference $((\mathrm{SMD}-0.17$ (95\% CI -0.55 to 0.38$\left.)), \mathrm{I}^{2} 0 \%\right)^{15}{ }^{16}$ between both interventions (online supplementary file 5). One study reported on long-term pain and function and found no difference $^{15}$ (online supplementary file 3 ). One study reported self-reported recovery at short term and found a significant effect (OR 2.03 (95\% CI 1.35 to 3.06)) in favour of prefabricated orthosess. ${ }^{20}$ 
Table 1 Overview risk of bias for the included studies

\begin{tabular}{|c|c|c|c|c|c|c|c|c|c|c|c|c|c|}
\hline Author & 1 & 2 & 3 & 4 & 5 & 6 & 7 & 8 & 9 & 10 & 11 & 12 & Total \\
\hline Al Bluwi et al $2011^{26}$ & $\mathrm{U}$ & $\mathrm{U}$ & U & $\mathrm{N}$ & $\mathrm{N}$ & $\mathrm{N}$ & $Y$ & U & $Y$ & Y & N & Y & 4 \\
\hline Baldassin et al $2009^{16}$ & $\mathrm{Y}$ & U & U & $Y$ & N & Y & U & U & Y & $Y$ & Y & $Y$ & 7 \\
\hline Caselli et al $1997^{36}$ & U & U & U & U & U & U & U & U & $\mathrm{N}$ & Y & $N$ & $\mathrm{~N}$ & 1 \\
\hline Dimou et al $2004^{28}$ & $\mathrm{Y}$ & U & U & $\mathrm{N}$ & $N$ & Y & U & U & $\mathrm{Y}$ & Y & $\mathrm{Y}$ & $\mathrm{Y}$ & 5 \\
\hline $\begin{array}{l}\text { Abd El Salem et al } \\
2011^{31}\end{array}$ & U & U & U & $\mathrm{N}$ & Y & $N$ & Y & U & $Y$ & Y & $Y$ & $Y$ & 6 \\
\hline Kavros $2005^{22}$ & U & U & U & $\mathrm{N}$ & $\mathrm{N}$ & U & $Y$ & U & Y & $Y$ & $Y$ & $Y$ & 5 \\
\hline Kriss $2003^{23}$ & U & U & U & $\mathrm{N}$ & $\mathrm{N}$ & $N$ & U & U & $\mathrm{Y}$ & Y & Y & $N$ & 3 \\
\hline Landorf et al $2006^{15}$ & $\mathrm{Y}$ & $\mathrm{Y}$ & U & Y & U & Y & Y & U & Y & Y & Y & Y & 9 \\
\hline Martin et al $2001^{37}$ & U & U & Y & $N$ & $\mathrm{~N}$ & $\mathrm{~N}$ & U & $N$ & $N$ & $Y$ & U & $\mathrm{N}$ & 2 \\
\hline Oliveira et al $2015^{19}$ & $\mathrm{Y}$ & $Y$ & $\mathrm{~N}$ & Y & $\mathrm{N}$ & $\mathrm{Y}$ & Y & U & Y & Y & $\mathrm{Y}$ & $\mathrm{Y}$ & 9 \\
\hline Pfeffer et al $1999^{20}$ & U & U & $\mathrm{Y}$ & N & $\mathrm{N}$ & $\mathrm{N}$ & Y & U & Y & Y & Y & $\mathrm{Y}$ & 6 \\
\hline Rome et al $2004^{29}$ & U & U & U & $\mathrm{N}$ & $\mathrm{N}$ & $\mathrm{N}$ & Y & U & $N$ & Y & U & $Y$ & 3 \\
\hline Roos et al $2006^{32}$ & Y & Y & U & N & $\mathrm{N}$ & N & U & $Y$ & Y & Y & Y & Y & 7 \\
\hline $\begin{array}{l}\text { Sharma and Loudon } \\
2010^{24}\end{array}$ & $Y$ & Y & $\mathrm{U}$ & N & $\mathrm{N}$ & $Y$ & Y & U & Y & Y & N & Y & 7 \\
\hline Turlik et al $1999^{25}$ & U & U & $N$ & $\mathrm{~N}$ & $\mathrm{~N}$ & $U$ & $N$ & U & Y & Y & N & $\mathrm{Y}$ & 3 \\
\hline Vicenzino et al $2015^{34}$ & $\mathrm{Y}$ & $\mathrm{Y}$ & $\mathrm{Y}$ & $\mathrm{N}$ & $\mathrm{N}$ & Y & U & U & Y & Y & $\mathrm{Y}$ & $Y$ & 8 \\
\hline Walther et al $2013^{30}$ & $\mathrm{Y}$ & U & $\mathrm{Y}$ & N & $\mathrm{N}$ & N & Y & $\mathrm{Y}$ & Y & Y & N & $\mathrm{Y}$ & 7 \\
\hline Winemiller et al $2003^{27}$ & $\mathrm{Y}$ & $Y$ & $\mathrm{~N}$ & Y & $\mathrm{Y}$ & $\mathrm{Y}$ & $\mathrm{Y}$ & $\mathrm{Y}$ & Y & Y & $\mathrm{Y}$ & $\mathrm{Y}$ & 11 \\
\hline Wrobel et al $2015^{21}$ & $Y$ & Y & $Y$ & Y & $N$ & Y & Y & U & Y & Y & N & Y & 9 \\
\hline Yucel et al $2013^{33}$ & $\mathrm{Y}$ & $\mathrm{Y}$ & $\mathrm{Y}$ & $\mathrm{N}$ & $\mathrm{N}$ & Y & $\mathrm{N}$ & U & Y & Y & $\mathrm{N}$ & $\mathrm{Y}$ & 7 \\
\hline
\end{tabular}

Criteria items: 1. Was the method of randomisation adequate? 2. Was the treatment allocation concealed? 3. Were the groups similar at baseline regarding the most important prognostic indicators? 4 . Was the patient blinded to the intervention? 5 . Was the care provider blinded to the intervention? 6 . Was the outcome assessor blinded to the intervention? 7. Were co-interventions avoided or similar? 8. Was the compliance acceptable in all groups? 9. Was the drop-out rate described and acceptable? 10. Was the timing of the outcome assessment similar in all groups? 11. Were all randomised participants analysed in the group to which they were allocated? 12 . Are reports of the study free of suggestion of selective outcome reporting?

\section{Custom-made versus sham orthoses}

Three studies compared sham orthoses with custom-made orthoses. $^{151921}$ All three studies had a low risk of bias. Data on short-term pain were pooled for all three studies, and no significant difference was found ((SMD 0.22 (95\%CI -0.05 to $0.50)) \mathrm{I}^{2} 0 \%$ ) between sham and custom-made orthoses (online supplementary file 6). One study reported pain at midterm and no difference was found between both interventions. ${ }^{19}$ One study reported pain at long term and no difference was found ${ }^{15}$ (online supplementary file 3 ). Three studies reported on function at short term and pooled results from two studies showed no significant difference ((SMD -0.27 (95\%CI -0.58 to 0.04$)$ ), $\mathrm{I}^{2} 0 \%$ ) between sham and custom-made orthoses(online supplementary file 7). Similar results were found in the study that could not be pooled for short-term function. ${ }^{21}$ One study reported on midterm function and found no difference between both interventions. ${ }^{19}$ One study reported on function at long term and found no significant difference ${ }^{15}$ (online supplementary file 3 ).

\section{Prefabricated versus sham orthoses}

Two studies compared a sham orthosis with a prefabricated orthosis. ${ }^{1521}$ Both studies had a low risk of bias. Pooled results on short-term pain showed no significant difference between prefabricated and sham orthoses (SMD 0.26 (95\%CI -0.09 to 0.60$)$ ), $\mathrm{I}^{2} 0 \%$ ) (online supplementary file 8 ). One study reported on long-term pain and found no difference ${ }^{15}$ (online supplementary file 3). Function was reported in both studies, but pooling was not possible due to incomplete reporting of the SD. ${ }^{21}$ Landorf $e t a l^{15}$ showed a significantly larger improvement in function at short term for prefabricated orthoses compared with sham orthoses (MD 8.40 (scale 0-100) (95\% CI 1.00 to $15.80)$ ), but no difference was seen at long term. Wrobel et al ${ }^{21}$ found no significant difference between the two interventions for the Foot Function Index (FFI) at short term ${ }^{21}$ (online supplementary file 3 ).

\section{Real versus sham orthoses}

Since no differences were found between custom-made and prefabricated orthoses, we pooled the custom-made and prefabricated orthoses together into one group called real orthoses and compared this group with sham orthoses. Three studies compared a real orthosis with a sham orthosis. ${ }^{15} 1921$ All studies had a low risk of bias. Pooled results on short-term pain showed no significant difference between real and sham orthoses (SMD - 0.22 (95\%CI -0.47 to 0.03 ), $\mathrm{I}^{2} 0 \%$ ) (online supplementary file 9). Data for function could be pooled from two studies. ${ }^{15} 19$ Pooled results on short-term function showed no significant difference between real and sham orthoses (SMD 0.23 (95\%CI -0.06 to $0.51), I^{2} 0 \%$ ) (online supplementary file 10 ).

\section{Over-the-counter arch support versus tension night splint}

One study with a high risk of bias compared an over-the-counter available arch support with a tension night splint. ${ }^{37}$ No significant differences were found between the two interventions in this study (online supplementary file 3 ).

\section{Custom-made orthoses versus tension night splint}

Two studies compared a custom-made orthosis with a tension night splint. ${ }^{32} 37$ Data from these studies could not be pooled. Martin $e t \mathrm{al}^{37}$ (high risk of bias) found no significant differences between the treatment groups for pain at short term (online supplementary file 3 ). ${ }^{37}$ Roos et al ${ }^{32}$ (low risk of bias) found no differences between the groups in pain at short term 
and midterm ${ }^{32}$ (online supplementary file 3 ). At long term, the orthosis group reported a significantly higher percentage of pain reduction compared with the tension splint group (62\% vs $48 \%, \mathrm{P}<0.01)$. No significant differences were found between the groups for function in daily living at short term and long term (online supplementary file 3). No significant difference was found between the groups for function in sport and recreation at short term and long term (online supplementary file 3). This difference was statistically significant at midterm (MD 21.00 (scale 0-100) (95\% CI 3.02 to 38.97$)$ ).

\section{Medial arch support versus low dye taping}

One study with a low risk of bias compared a medial arch support with low dye taping. ${ }^{31}$ Patients in both groups additionally received physical therapy and ultrasound treatment. Significant differences were found on both pain (Visual Analogue Scale (VAS)) and function (Manchester Foot Pain and Disability Schedule) at short term in favour of the medial arch support group (MD 1.67 (scale $0-10)$ (95\% CI 0.58 to 2.77) and MD 5.00 (scale $0-51$ ) (95\% CI 1.50 to 8.51$)$ ), respectively).

\section{Prefabricated foot insert versus ankle brace}

The effect of a prefabricated foot insert was compared with the effect of an ankle brace in one study with a high risk of bias. ${ }^{22}$ Additionally, both groups were instructed to perform stretching exercises and to wear athletic footwear as much as possible. No significant differences were found in this study(online supplementary file 3 ).

\section{Orthoses versus corticosteroid injection}

The effect of an anti-pronatory pad was compared with the effect of corticosteroid injection in one study with a high risk of bias. ${ }^{23}$ The groups treated with injection had a significantly lower VAS score at short term (MD 28.70 (scale 0-100) $(95 \%$ CI 19.53 to 37.87)) This effect was no longer seen at midterm(online supplementary file 3 ). The effect of a prefabricated orthosis was compared with the effect of a ultrasound-guided corticosteroid injection in one study with a low risk of bias. ${ }^{33}$ The Foot and Ankle Outcome Score pain subscore was significantly better in the injection group at the short-term follow-up (MD -6.05 (scale $0-100)$ (95\% CI 0.66 to 11.42$)$ ).

\section{Orthoses versus stretching exercises}

The effect of stretching exercises of the Achilles and plantar fascia were compared with different orthoses(felt full-length prefabricated orthoses, rubber heel cup, silicone heel cup and custom-made full-length orthoses) in one study with a low risk of bias. ${ }^{20}$ Only the group treated with the silicone heel cup showed significant larger improvement in the self-reported recovery rate at short term compared with the group performing stretching exercises (OR 7.79 (95\%CI 3.85 to 15.84 )). No differences were found in self-reported recovery between the other interventions (online supplementary file 3). No differences in the FFI pain score at short term were found when comparing stretching exercises with the orthoses (online supplementary file 3 ). The effect of a static ankle brace was compared with the effect of stretching exercises in one study with a low risk of bias. ${ }^{24}$ No differences between the different interventions were found in this study (online supplementary file 3 ).

\section{Custom-made foot orthoses versus heel cup}

The effect of a heel cup was compared with the effect of a custommade orthosis in two studies. ${ }^{20} 25$ One study with a low risk of bias compared rubber and silicone heel cups with a full-length custom-made orthosis and found no differences between the groups $^{20}$ (online supplementary file 3 ). The effect of a custommade orthosis was compared with urethane heel pads in one study with a high risk of bias. ${ }^{25}$ A significantly lower pain score at midterm was seen in the custom-made orthosis group (VAS score morning pain $\mathrm{P}=0.0042$, VAS score rest of life $\mathrm{P}=0.009$ ).

\section{EZStep versus physiotherapy and injection}

The effect of EZStep was compared with physiotherapy alone and with physiotherapy and injection in one study with a high risk of bias. ${ }^{26}$ At midterm, a significantly lower VAS score was seen in the EZStep group compared with the physiotherapy group (MD -4.63 (scale $0-10)(95 \% \mathrm{CI}-5.65$ to -3.61$)$ ) and with the physiotherapy and injection group ( $\mathrm{MD}-2.83$ (scale $0-10)(95 \%$ CI -3.45 to -0.55$))$.

\section{Prefabricated orthoses versus magnetic orthoses}

The effect of a prefabricated orthosis was compared with an orthosis with magnetic properties in two studies. ${ }^{27} 36$ One study with a high risk of bias compared a prefabricated orthosis with the same orthosis, but containing a magnetic foil. ${ }^{36}$ No differences were found at short term (online supplementary file 3 ). One study with a low risk of bias compared cushioned orthoses containing either a real or sham magnet and found no differences at short term ${ }^{27}$ (online supplementary file 3 ).

\section{Custom-made orthoses versus chiropractic manipulation plus stretching exercises}

The effect of custom orthoses was compared with chiropractic manipulation in one study with a high risk of bias. ${ }^{28}$ No differences were found between the two groups for most outcomes at short term. Except for the Numeric Rating Scale (NRS) for worst pain at 15 days, a more favourable effect of manipulation and stretching was found $(\mathrm{P}=0.03)$.

\section{Prefabricated orthoses versus heel cup}

The effect of rubber and silicone heel cups was compared with a prefabricated orthosis in one study with a low risk of bias. ${ }^{20}$ At short term, no differences were found between the groups for pain (online supplementary file 3). A statistically significant difference was found between the prefabricated orthosis and the silicone heel cup regarding self-reported response (OR 0.21 (95\%CI 0.07 to 0.59$)$ ). No difference was found between the prefabricated orthosis and the rubber heel cup (online supplementary file 3 ).

\section{Prefabricated orthoses versus another type of prefabricated orthosis}

A non-supportive orthosis, a soft foam orthosis and a foam covered rigid self-supporting orthosis were compared in one study with a low risk of bias. ${ }^{30}$ There was no difference in average pain score (VAS) at short term between the soft foam orthosis and the rigid self-supporting orthosis (online supplementary file 3). A functional orthosis was compared with an accommodative orthosis in one study with a high risk of bias. ${ }^{29}$ No significant differences were found (online supplementary file 3).

\section{Orthoses versus different types of footwear}

A prefabricated orthosis, a contoured sandal and a flat flip flop were compared in one study with a low risk of bias. ${ }^{34}$ A significantly higher number of patients with orthosis had a Lower Extremity Functional Score (LEFS) of 9 or higher compared with 
the flip flop at short term (OR 0.48 (95\%CI 0.27 to 0.85$)$ ), but no difference was found in function (LEFS) when the orthosis was compared with the sandal. No difference was found in the number of patients with a Foot and Ankle Ability Measure of 8 or higher at short term when comparing the groups (online supplementary file 3 ).

\section{DISCUSSION}

This systematic review and meta-analysis shows that orthosis interventions are not superior in improving pain, function or self-reported recovery when compared with other conservative interventions in patients with PHP. These findings extend the findings of a previous Cochrane review focusing on custommade orthoses. ${ }^{17}$ A recently published systematic review by Whittaker et al found a small effect in favour of real orthoses compared with sham orthoses. ${ }^{38}$ The difference in findings can be explained by the different definitions for short-term, midterm and long-term follow-up. In our review, we defined short term as 0 up to 3 months, midterm as 3 to 12 months and long term was defined as more than 12 months. We chose these definitions because they are commonly applied definitions in the literature and they reflect the long-term complaints of some patients with PHP experience. Patients with complaints for over 12 months are considered chronic or recalcitrant. ${ }^{39-42}$

\section{What type of orthosis is best?}

A total of 20 articles studied the effect of eight different types of orthoses, that is, custom-made orthosis, ${ }^{15} 1619-2125283237$ prefabricated orthosis, ${ }^{15}{ }^{26} 20-22$ 29-31 333437 EZ Step, ${ }^{26}$ orthosis with magnetic properties, ${ }^{27} 36$ heel cup,${ }^{20} 25$ static ankle brace, ${ }^{24}$ Airheel Cast ${ }^{22}$ and an antipronatory pad. ${ }^{23}$ The studies also differed in time of follow-up, outcome measures and control interventions applied. For this reason, pooling of data was often not possible.

We were, however, able to pool data from six different studies, comparing custom-made orthoses, prefabricated orthoses and sham orthoses. ${ }^{15} 16$ 19-21 37 The narrow CIs around the pooled estimates imply a relatively precise estimate of the effects found. Although most of the included individual studies reported no differences in the effectiveness of the different interventions, 10 studies found a statistically significant difference on one of the outcome measures at a certain time point. ${ }^{15} 202325262831-34$ Most of those significant differences were not clinically relevant as point estimates were small compared with the minimal important difference. Landorf et al compared a commonly used prefabricated orthosis with a sham device and found a significant difference in function score of the Foot Health Status Questionnaire (FHSQ) at 3 months of 8.40 on a scale of 0 to 100 which in more than the minimal important difference of seven points, but no differences were found at long-term follow-up. ${ }^{153}$ Other studies only found differences on subscales of an outcome or had methodological issues. ${ }^{26} 3132$ Three studies found small non-significant differences favouring customised orthotics. Even though the CIs indicated that these were close to significance, it is unlikely that the differences would be clinically meaningful due to their size. ${ }^{15} 1937$

A large proportion of the included studies had a relatively small sample size and 8 out of 20 had a high risk of bias. Only three studies used a sham or placebo device as a control which illustrates the difficulty in blinding patients for the type of orthosis. Sham orthoses might have some therapeutic effect as well because they are often designed to resemble the real orthosis intervention as much as possible and therefore also provide some mechanical effect. ${ }^{445}$ The different approaches for the selection or prescription of foot orthoses used in the various studies may not reflect clinical practice.

Only a few studies note whether or not a clinician was consulted during the trial. ${ }^{15} 2126-3037$ This may also reflect the lack of consensus in clinical practice on the treatment approach for PHP with orthoses. Additionally, in clinical trials, orthoses are prescribed to all patients randomised to the intervention group, while in clinical practice other treatment options are often also considered; the indication for orthoses depends on various variables, including patient preference and costs, and therefore the socioeconomic status of the patient. The clinician will also consider how likely a patient is to adhere to treatment based on their experience. A clear advantage of orthoses is that they do not require as much effort by the patient to adhere to the treatment compared with exercises or stretching. These challenges make the design of trials for orthotic treatment difficult. Nevertheless, RCTs with a large sample size using a valid placebo or sham intervention are needed to be able to draw firm conclusions on the effectiveness of commonly used orthoses in clinical practice.

\section{Natural history of PHP: influence on analysis}

Most studies reported a significant improvement over time in patients treated with orthoses as well as patients treated with other conservative interventions. This effect is likely explained by the favourable clinical course of PHP. Previous literature has reported a complete remission of symptoms after 12 months in $80 \%$ of patients, treated with varying interventions. ${ }^{69}$ Other literature found that $>40 \%$ of patients still have complaints after 2 years. ${ }^{10}$ The included studies in this review reported recovery rates at short term ranging from 10\% up to $99 \%$. ${ }^{19} 2025273436$ Orthoses are thought to shorten the time until pain relief. We only observed small differences at short and mid-term follow-up when the orthoses were compared with other conservative interventions. Two studies that compared an orthosis with corticosteroid injection reported that the injection was superior to the orthosis in the short term. ${ }^{23}{ }^{33}$ However, we found no evidence for this for the long term and there are putative negative effects of corticosteroid injections on long-term outcomes. ${ }^{46}{ }^{47}$ As literature on the clinical course of complaints without treatment ('wait and see') is lacking, and none of the included studies compared orthoses with 'no treatment', the additive value of orthoses remains largely unclear.

\section{STRENGTHS, LIMITATIONS AND CONCLUSION}

Our study provides a major update on the field since the 2008 Cochrane review, 'Custom-made foot orthoses for the treatment of foot pain'. ${ }^{17}$ Additionally, we have included more types of orthoses in our review. We used rigorous methods in the search strategy, data analysis and risk of bias elements of the systematic review. $^{48}$

Limitations of this review include the heterogeneity between the included studies in types of interventions applied, outcome measures and timing of follow-up. This made data pooling difficult. We have therefore rescaled outcome measures where possible and applied predefined timeframes for short, mid and long-term follow-up. The highest $\mathrm{I}^{2}$ found in the analyses performed was $20 \%$, which implies little statistical heterogeneity and therefore pooling seemed justified. We also used a broad definition of PHP when searching for relevant articles. It is possible that the included articles studied different subcategories of PHP. Since the terminology of PHP is subject to discussion and because the terms used to describe this condition have changed in recent times, using a 
more narrow definition might lead to the incorrect excluding of relevant articles. ${ }^{49}$

In summary, foot orthoses are not superior for improving pain and function compared with sham or other orthoses, or other conservative interventions in patients with PHP. We conclude that clinicians should be reserved in prescribing foot orthoses in all patients with PHP and take factors like patient preference and adherence into account.

\section{What is already known?}

Foot orthoses are often applied in the treatment of plantar heel pain despite a lack of evidence.

\section{What are the new findings?}

- There was no difference in improvement in pain or function between prefabricated, custom-made and sham orthoses in the treatment of patients with plantar heel pain.

- A significant number of studies studying foot orthoses in patients with plantar heel pain have methodological limitations.

Acknowledgements The author would like to thank Gerdien B. de Jonge and Wichor M. Bramer, Biomedical Information Specialist(s) Medical Library Erasmus MC, for their support in the literature search.

Contributors All five authors made a substantial contribution to the design the drafting and the revising of the manuscript and have read and approved the final version. None of the authors has a direct or indirect commercial financial incentive associated with publishing this article. All five authors agree to be accountable for all aspects of the work to ensure that questions related to the accuracy or integrity of the work are appropriately resolved.

Funding This research received no specific grant from any funding agency in the public, commercial or not-for-profit sectors.

Competing interests None declared.

Provenance and peer review Not commissioned; externally peer reviewed.

(c) Article author(s) (or their employer(s) unless otherwise stated in the text of the article) 2018. All rights reserved. No commercial use is permitted unless otherwise expressly granted.

\section{REFERENCES}

1 Riddle DL, Schappert SM. Volume of ambulatory care visits and patterns of care for patients diagnosed with plantar fasciitis: a national study of medical doctors. Foot Ankle Int 2004;25:303-10.

2 Riddle DL, Pulisic M, Pidcoe P, et al. Risk factors for Plantar fasciitis: a matched casecontrol study. J Bone Joint Surg Am 2003;85-A:872-7.

3 Scher DL, Belmont PJ, Bear R, et al. The incidence of plantar fasciitis in the United States military. J Bone Joint Surg Am 2009;91:2867-72.

4 Thomas JL, Christensen JC, Kravitz SR, et al. The diagnosis and treatment of heel pain: a clinical practice guideline-revision 2010. J Foot Ankle Surg 2010:49(3 Suppl):S1-S19.

5 Taunton JE, Ryan MB, Clement DB, et al. A retrospective case-control analysis of 2002 running injuries. Br I Sports Med 2002;36:95-101.

6 Buchbinder R. Clinical practice. Plantar fasciitis. N Eng/ J Med 2004:350:2159-66.

7 Irving DB, Cook JL, Young MA, et al. Impact of chronic plantar heel pain on healthrelated quality of life. J Am Podiatr Med Assoc 2008;98:283-9.

8 Beeson P. Plantar fasciopathy: revisiting the risk factors. Foot Ankle Surg 2014;20:160-5.

9 Covey CJ, Mulder MD. Plantar fasciitis: How best to treat? J Fam Pract 2013:62:466-71.

10 Digiovanni BF, Nawoczenski DA, Malay DP, et al. Plantar fascia-specific stretching exercise improves outcomes in patients with chronic plantar fasciitis. A prospective clinical trial with two-year follow-up. J Bone Joint Surg Am 2006:88:1775-81.

11 Orchard J. Plantar fasciitis. BMJ 2012;345:e6603.

12 Cutts S, Obi N, Pasapula C, et al. Plantar fasciitis. Ann R Coll Surg Engl 2012;94:539-42.

13 Rathleff MS, Mølgaard CM, Fredberg U, et al. High-load strength training improves outcome in patients with plantar fasciitis: A randomized controlled trial with 12-month follow-up. Scand J Med Sci Sports 2015;25:e292-e300.
14 Rompe JD. Plantar fasciopathy. Sports Med Arthrosc 2009;17:100-4.

15 Landorf KB, Keenan AM, Herbert RD. Effectiveness of foot orthoses to treat plantar fasciitis: a randomized trial. Arch Intern Med 2006;166:1305-10.

16 Baldassin V, Gomes CR, Beraldo PS. Effectiveness of prefabricated and customized foot orthoses made from low-cost foam for noncomplicated plantar fasciitis: a randomized controlled trial. Arch Phys Med Rehabil 2009:90:701-6.

17 Hawke F, Burns J, Radford JA, et al. Custom-made foot orthoses for the treatment of foot pain. Cochrane Database Syst Rev 2008:3:CD006801.

18 van Tulder MW, Suttorp M, Morton S, et al. Empirical evidence of an association between internal validity and effect size in randomized controlled trials of low-back pain. Spine 2009;34:1685-92

19 Oliveira HA, Jones A, Moreira E, et al. Effectiveness of total contact insoles in patients with plantar fasciitis. J Rheumatol 2015:42:870-8.

20 Pfeffer G, Bacchetti P, Deland J, et al. Comparison of custom and prefabricated orthoses in the initial treatment of proximal plantar fasciitis. Foot Ankle Int 1999;20:214-21

21 Wrobel JS, Fleischer AE, Crews RT, et al. A randomized controlled trial of custom foot orthoses for the treatment of plantar heel pain. J Am Podiatr Med Assoc 2015:105:281-94.

22 Kavros SJ. The efficacy of a pneumatic compression device in the treatment of plantar fasciitis. J App/ Biomech 2005;21:404-13.

23 Kriss S. Injectable steroids in the management of heel pain. A prospective randomised trial. British Journal of Podiatry 2003;62:40-3.

24 Sharma NK, Loudon JK. Static progressive stretch brace as a treatment of pain and functional limitations associated with plantar fasciitis: a pilot study. Foot Ankle Spec 2010:3:117-24.

25 Turlik MA, Donatelli TJ, Veremis MG. A comparison of shoe inserts in relieving mechanical heel pain. Foot 1999:9:84-7.

26 Al-Bluwi MT, Sadat-Ali M, Al-Habdan IM, et al. Efficacy of EZStep in the management of plantar fasciitis: a prospective, randomized study. Foot Ankle Spec 2011:4:218-21.

27 Winemiller MH, Billow RG, Laskowski ER, et al. Effect of magnetic vs sham-magnetic insoles on plantar heel pain: a randomized controlled trial. JAMA 2003;290:1474-8.

28 Dimou ES, Brantingham JW, Wood T. A randomized, controlled trial (with blinded observer) of chiropractic manipulation and achilles stretching vs orthotics for the treatment of plantar fasciitis. J Am Chiropr Assoc 2004:41:32-42.

29 Rome K, Gray J, Stewart F, et al. Evaluating the clinical effectiveness and costeffectiveness of foot orthoses in the treatment of plantar heel pain: a feasibility study. J Am Podiatr Med Assoc 2004;94:229-38.

30 Walther M, Kratschmer B, Verschl J, et al. Effect of different orthotic concepts as first line treatment of plantar fasciitis. Foot Ankle Surg 2013;19:103-7.

31 Abd El Salam MS, Abd Elhafz YN. Low-dye taping versus medial arch support in managing pain and pain-related disability in patients with plantar fasciitis. Foot Ankle Spec 2011:4:86-91.

32 Roos E, Engström M, Söderberg B. Foot orthoses for the treatment of plantar fasciitis. Foot Ankle Int 2006;27:606-11.

33 Yucel U, Kucuksen S, Cingoz HT, et al. Full-length silicone insoles versus ultrasoundguided corticosteroid injection in the management of plantar fasciitis: a randomized clinical trial. Prosthet Orthot Int 2013:37:471-6.

34 Vicenzino B, McPoil TG, Stephenson A, et al. Orthosis-shaped sandals are as efficacious as in-shoe orthoses and better than flat sandals for plantar heel pain: a randomized control trial. PLoS One 2015;10:0142789.

35 Bishop C, Thewlis D, Hillier S. The effect of custom foot orthotics and footwear on firststep pain in people with plantar heel pain: a pragmatic randomized controlled trial. J Sci Med Sport 2015;19:e24.

36 Caselli MA, Clark N, Lazarus S, et al. Evaluation of magnetic foil and PPT Insoles in the treatment of heel pain. J Am Podiatr Med Assoc 1997;87:11-16.

37 Martin JE, Hosch JC, Goforth WP, et al. Mechanical treatment of plantar fasciitis. A prospective study. J Am Podiatr Med Assoc 2001;91:55-62.

38 Whittaker GA, Munteanu SE, Menz HB, et al. Foot orthoses for plantar heel pain: a systematic review and meta-analysis. Br I Sports Med 2017:bjsports-2016-097355.

39 Wheeler PC, Tattersall C. Extracorporeal shockwave therapy plus rehabilitation for patients with chronic plantar fasciitis might reduce pain and improve function but still not lead to increased activity: a case-series study with multiple outcome measures. J Foot Ankle Surg 2017

40 Rizk AS, Kandel WA, Tabl EAE, et al. Mid-sole release of the plantar fascia combined with percutaneous drilling of the calcaneus for treatment of resistant heel pain. Foot Ankle Int 2017;38:1271-7.

41 Kumai T, Samoto N, Hasegawa A, et al. Short-term efficacy and safety of hyaluronic acid injection for plantar fasciopathy. Knee Surg Sports Traumatol Arthrosc 2017.

42 Scheuer R, Friedrich M, Hahne J, et al. Approaches to optimize focused extracorporeal shockwave therapy (ESWT) based on an observational study of 363 feet with recalcitrant plantar fasciitis. Int J Surg 2016;27:1-7.

43 Landorf KB, Radford JA, Hudson S, et al. Minimal Important Difference (MID) of two commonly used outcome measures for foot problems. J Foot Ankle Res 2010:3:7.

44 Crosbie J, Burns J. Predicting outcomes in the orthotic management of painful, idiopathic pes cavus. Clin I Sport Med 2007;17:337-42

45 Hawke F, Burns J. Understanding the nature and mechanism of foot pain. J Foot Ankle Res 2009;2:1. 
46 Ang TW. The effectiveness of corticosteroid injection in the treatment of plantar fasciitis. Singapore Med J 2015;56:423-32.

47 David JA, Sankarapandian V, Christopher PR, et al. Injected corticosteroids for treating plantar heel pain in adults. Cochrane Database Syst Rev 2017;6:CD009348.
48 Ardern CL. Systematic review hacks for the sports and exercise clinician: five essential methodological elements. Br J Sports Med 2016;50:447-9.

49 Riel H, Cotchett M, Delahunt E, et al. Is 'plantar heel pain' a more appropriate term than 'plantar fasciitis'? Time to move on. Br J Sports Med 2017;51:1576-7. 